\title{
Distributed Multiuser Sequential Channel Sensing Schemes in Multichannel Cognitive Radio Networks
}

\author{
Hossein Shokri-Ghadikolaei, Student Member, IEEE, Fatemeh Sheikholeslami, \\ and Masoumeh Nasiri-Kenari, Member, IEEE
}

\begin{abstract}
Effective spectrum sensing strategies enable cognitive radios (CRs) to identify and opportunistically transmit on under-utilized spectral resources. In this paper, sequential channel sensing problems for single and multiple secondary users (SUs) networks are effectively modeled through finite state Markovian processes. More specifically, a model for single user case is introduced and its performance is validated through analytical analysis. Then, in order to address multiple SUs case, this model is extended to include the modified p-persistent access (MPPA) protocol. Since the scheme utilized experiences a high level of collision among the SUs, to mitigate the problem appropriately, p-persistent random access (PPRA) protocol is considered, which offers higher average throughput for SUs by statistically distributing their loads among all channels. The structure and performance of the proposed schemes are discussed in detail, and a set of illustrative numerical results is presented to validate and compare the performance of the proposed senseaccess strategies.
\end{abstract}

Index Terms-Cognitive radio, spectrum handover, sequential channel sensing, queuing networks, secondary user's throughput.

\section{INTRODUCTION}

$\mathbf{E}$ MERGING new wireless applications and ever-growing needs for a higher data rate have incredibly increased the demand for accessing to spectrum in the past ten years. Though the available spectrum resources seem to not meet the ever-growing demand, many investigations reveal that the spectrum is grossly under-utilized in time, space, and other dimensions [1]. In order to mitigate the spectrum scarcity problem, cognitive radio (CR) concept, as a potential communication paradigm, has been introduced to improve spectrum efficiency by allowing low-priority secondary users (SUs) to opportunistically exploit the unused licensed spectrums of high-priority primary users (PUs), called white spaces, in a somewhat intelligent manner [2]. To this end, first these temporarily-available transmission opportunities must be explored through appropriate and reliable spectrum sensing schemes. Limited number of possible observations and dynamic nature of observed signals lead to imperfect sensing which is usually described by false alarm and miss detection probabilities. An SU should stop its transmission and vacate the occupied channel, whenever the corresponding PU has data

Manuscript received March 13, 2012; revised October 8, 2012 and January 25, 2013; accepted February 15, 2013. The associate editor coordinating the review of this paper and approving it for publication was X. Ma.

The authors are with the Wireless Research Laboratory, Electrical Engineering Department, Sharif University of Technology, Tehran, Iran (e-mail: \{hshokri, sheikholeslami\}@ee.sharif.edu, mnasiri@sharif.edu).

Digital Object Identifier 10.1109/TWC.2013.032113120359 to transmit on this channel. To provide a reliable transmission for the SUs, a set of procedures called spectrum handover (SHO) is initiated to help the SU to effectively find a new transmission opportunity and resume its unfinished transmission [1]- [3]. Average SUs' throughput and average consumed energy for sensing process are two important performance metrics, which directly depend on the candidate primary channels for sensing and transmission, and are evaluated in order to compare various channel sensing-access policies.

Generally speaking, there exists more than one channel to be sensed by a CR. To deal with this fact, sensing schemes are commonly divided into two categories, i.e., wideband sensing and narrowband sensing. Sensing is considered wideband when multiple channels are sensed simultaneously. On the other hand, when only one channel is sensed at a time, the sensing process is called narrowband. Easy implementation, lower power consumption, and less computational complexity lead to great interest in narrowband sensing. The handover process, associated with narrowband sensing, is mainly categorized into reactive and proactive policies. In the proactivedecision schemes, long term traffic statistics of the channels are exploited in order to establish a proper channel sequence for future handovers [4]. This sequence determines the target channels which an SU should transmit on. However, a great delay on the SU connection is imposed by these schemes; because the predetermined target channel may not be available for a long time, when an SHO is requested. On the other hand, in the reactive-based SHO strategies, the SU searches among channels to find a free channel and pursues its unfinished transmissions [5]. In this category, the SU does not wait until the end of the corresponding PU's transmission, and tries to find a free channel by sensing new channels sequentially according to a predetermined set of channels called sensing sequence (SS), although a longer switching delay becomes inevitable. A comprehensive comparison of these two SHO schemes is provided in [6]. In this paper, we focus on the modeling technique and performance analysis for the reactivebased SHO schemes.

In [7], a generalized reactive SHO scheme is proposed in which an SU chooses to stay on its current channel or initiate a search procedure to find a new free channel based on expected PU's load. Minimizing the average number of handovers through the concept of underlay transmission is investigated in [8]. In particular, the SUs decrease their transmission powers, if applicable, to avoid unnecessary handovers while meeting the quality of service requirements of the PUs. 
In [9], the authors consider sequential channel sensing for multichannel cognitive radio networks (CRNs), and derive the best set of candidate channels for data transmission. Optimal sensing scheduling policy is proposed in [10] in which the authors develop an analytical framework to determine which secondary users should sense which channels with what miss detection and false alarm probabilities. In [11], while adopting a periodic channel sensing with memoryless transmission (PSMT) policy, the problem of maximizing the SU's throughput under a tight collision constraint is inspected. In [12] and [13], the sequential channel sensing problems are formulated based on maximizing the throughput of the SUs. While in these works, the optimum sensing times have been studied, the effects of the sensing errors have not been addressed. Considering the effect of imperfect sensing, the authors of [14] have investigated the minimization of the average sensing time in a sequential channel sensing strategy. Maximizing the secondary network throughput through finding optimal channel sensing sequences is studied only for one SU in [15], for two SUs in [16], and for multiple SUs in [17], [18]. An analytical framework is developed in [19], which facilitates the performance evaluation of different multichannel multistage spectrum sensing schemes for a CRN with one SU. In [19], the average SU throughput and the average probability of collision between the SU and PU are considered as performance metrics. The authors in [20] present an analytical framework to compare different spectrum sensing-access policies. They focus on the throughput of the SUs as a comparative metric for the performance assessment, and propose a new spectrum sensing MAC protocol, called truncated time division multiple access.

Although the sequential channel sensing scheme exploited in this paper has been studied in the literature to some extents so far, some issues remain unsolved. As discussed briefly above, though in [15], [16], and [17], the authors have investigated the optimal sensing sequence in a singleuser, two-user, and multiuser secondary networks, respectively, the existence of a coordinator is an inseparable part of those centralized algorithms. The coordinator computes the optimal sensing sequences, and then let the SUs know their related sequences. Not only a centralized network cannot be assumed in many CRNs applications, but those proposals impose massive computational burden on the network as well. For instance, as shown in [17], the computational complexity of finding the optimal sensing sequences is in order of $\mathcal{O}\left(N_{p}^{N_{s} N_{p}}\right)$, which is not scalable regarding to the number of PUs $N_{p}$, and the number of SUs $N_{s}$. p-persistent MAC protocol has been introduced in [21] and [22] to solve the mentioned problems, in which the authors have applied the protocol to a multiuser network. In that scenario, SUs are allowed to sense only one channel at each time slot, and the channel occupancy of the PU is considered unknown and constant for a time duration, in which sensing takes place sequentially.

In contrast to those studies, in this paper, we propose a new Markovian-based analytical model, which enables us to model a fully distributed CRN, where the SUs cannot cooperate with each other. This assumption significantly reduces the bandwidth required for control channel, and therefore decreases the problems associated with control channel establishment in CRN terminology [23], [24].

As a first step, we consider a CRN with a single $\mathrm{SU}$ and introduce the finite state Markovian process-based structure to effectively model the SU's behavior. Besides providing a systematic approach for the performance evaluation, the model can be easily extended to the multiple SUs case by slightly modifying its nodes and routing probabilities. Before extending the proposed model to the multiple SUs case, we validate the model by directly computing the system throughput and comparing the result with the one obtained using the model. We call the extended model for the multiple SUs scenario as modified p-persisted access (MPPA). The performance of the MPPA scheme in terms of the average SUs' throughput and the average number of handovers is analyzed. After a discussion about the benefits and drawbacks of the MPPA scheme, the p-persistent random access (PPRA) policy is considered to appropriately mitigate the problem associated with the MPPA algorithm. In this model, the SUs sequentially sense channels with the same as well as various sensing orders. Specifically, by this policy, the loads of the SUs are distributed among all available channels, and hereby the CRN throughput increases through the reduction of the contention level among SUs. Finally, the SUs' throughput and the average number of handovers for the PPRA scheme are also derived.

The rest of this paper is organized as follows. In Section II, we describe the considered CR network, introduce the single SU based queuing model, and validate the model. In Section III, the model is extended for the multiple secondary user case, and then its performance is evaluated. In Section IV, the structure and the performance of the PPRA scheme are discussed in details. Numerical results are then presented in Section $\mathrm{V}$, followed by concluding remarks provided in Section VI.

\section{System Modeling And VAlidation}

\section{A. System Model}

We consider a time slotted CRN with $N_{s}$ SUs, which attempt to opportunistically transmit on the channels dedicated to the $N_{p}$ PUs. The SUs are synchronous in time-slots with other SUs as well as the PUs. The PUs start their transmissions only at the beginning of the slot whenever they have data for transmissions. Therefore, in order to find the transmission opportunities appropriately and to protect the PUs from a harmful interference, the SUs sense the channels at the beginning of each time-slot, by which the channels can be established as occupied or vacant. The SUs utilize narrowband spectrum sensing, i.e., they sense only one spectrum (out of $N_{p}$ spectrums) at a time. The secondary network is considered saturated meaning that the SUs always have packets to transmit; therefore they will start their transmissions when an opportunity is found. Each SU senses the channels according to its SS sequentially, i.e., the SU senses the first channel that is assigned to its SS for a predetermined time duration $\tau$ (channel sensing time), and then starts sensing the second channel if and only if the first channel is sensed busy. This procedure will be continued until a transmission opportunity is found. 


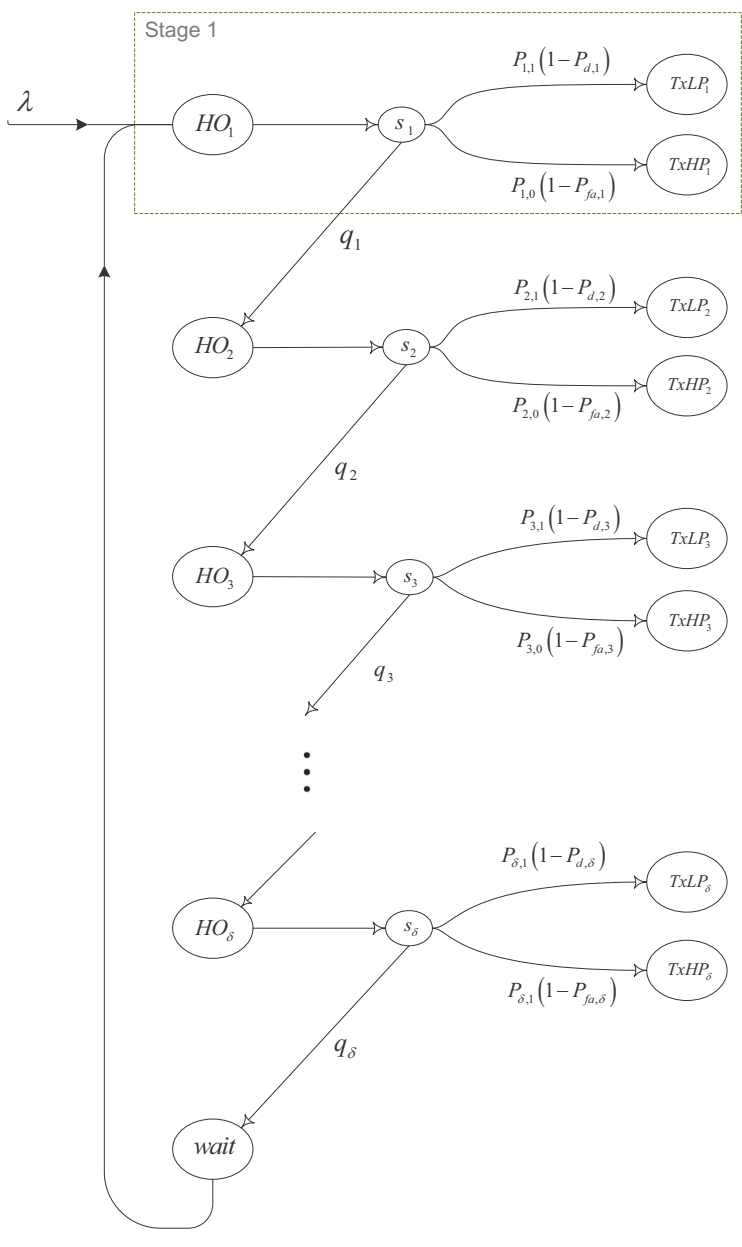

Fig. 1. The proposed model for single SU case.

In order to switch to a new channel, a secondary device needs a specific and constant time duration $\tau_{h o}$ to prepare its sensing circuitry. After sensing $(i-1)$ occupied channels, if the SU finds the $i$-th channel free, the user will transmit data on that channel until the end of the slot. In this case, the wasted time length, i.e., the time spent on the sensing and handover, is equal to $\tau+(i-1)\left(\tau+\tau_{h o}\right)$. If an SU starts transmitting on the $i$-th channel of its SS, the time length left in the slot for the transmission is,

$$
R T_{i}=T-\tau-(i-1)\left(\tau+\tau_{h o}\right)
$$

where $T$ is the time slot duration.

\section{B. Proposed Markov Chain}

In this part, we model the channel search and access policy for a CRN containing one SU by an open network [25] as depicted in Fig. 1. It will be extended to the multiuser case in the next sections. The network is comprised of several nodes corresponding to different stages of spectrum sensing and packet transmission. In the proposed model, the arrival of a request to the queuing network represents the transmission attempt of a packet including channel sensing and handovers. The request leaves the network after receiving the service from a subset of nodes.

Different handovers are modeled through nodes $\mathrm{HO}_{i}$, $i=1,2, \ldots, \delta$, where $\delta$ is the maximum number of allowed handovers and will be discussed later. It is worth noting that the first handover node does not really exist in the process of finding a transmission opportunity, and it appears just for providing a symmetry in the model. Let $S_{i}$ denote the sensing process of $i$-th channel. At the beginning of each time slot, an SU's request enters the node $\mathrm{HO}_{1}$ (in Fig. 1), and immediately is routed to the first sensing node, $S_{1}$. After $\tau$ time units, the channel sensor routes the request to the transmitter nodes (node $T x L P_{1}$ or $T x H P_{1}$ ) or to the second handover node, $H_{2}$. Let us define the $i$-th stage of the sensing-transmission process, shown in Fig. 1, as the set of nodes $H O_{i}, S_{i}, T x L P_{i}$, and $T x H P_{i}$. At the $i$-th stage, the $i$-th channel is sensed free if (a) the $i$-th PU is absence (with the probability of $P_{i, 0}$ ) and the SU correctly detects this transmission opportunity, with the probability of $P_{i, 0}\left(1-P_{f a, i}\right)$, or (b) the channel is occupied by the PU (with the probability of $P_{i, 1}=1-P_{i, 0}$ ) but the SU mistakenly senses this channel free, with the probability of $P_{i, 1}\left(1-P_{d, i}\right)$, where $P_{f a, i}$ and $P_{d, i}$ denote the false alarm and detection probabilities of the sensing process of the $i$-th channel. Please note that similar to [26], [27], in this study, we evaluate the SUs performance while their impacts on the PUs communications are limited, i.e., the admissible false alarm and miss detection probabilities are restricted according to the IEEE 802.22 standard [28]. Hence, the desired QoS of the PUs is provided. Nodes $T x H P_{i}$ model the first case (case a) in which the SU will be able to transmit on the $i$-th channel for the rest of the time slot with the capacity of $C_{0}=\log _{2}\left(1+\gamma_{s}\right)$. In the same way, in nodes $T x L P_{i}$ which models the later case (case b), the SU will be able to transmit on the $i$-th channel for the rest of the time slot with the capacity of $C_{1}=\log _{2}\left(1+\frac{P_{s}}{N_{0}+P_{p}}\right)=\log _{2}\left(1+\frac{\gamma_{s}}{1+\gamma_{p}}\right)$, where $\gamma_{s}$ and $\gamma_{p}$ are the received SNRs due to the secondary and primary users' signals at the SU receiver, respectively ${ }^{1}$. If none of those two cases occur, the request will be routed to the node $H O_{i+1}$ with the probability $q_{i}$,

$$
q_{i}=P_{i, 0} P_{f a, i}+P_{i, 1} P_{d, i}
$$

This procedure is continued until the maximum number of admissible handovers is reached. This parameter is limited by two constraints. First, the number of sensed channels cannot exceed the number of the PUs. Second, the elapsed time for both sensing and handover procedures cannot exceed the time slot duration, $T$. So, the maximum number of sensed channels is computed:

$$
\begin{aligned}
\delta & =1+\text { maximum number of admissible handovers } \\
& =1+\min \left(\left\lfloor\frac{T-\tau}{\tau+\tau_{h o}}\right\rfloor, N_{P}-1\right)
\end{aligned}
$$

After the $\delta$-th stage, the request is routed to node wait in order to wait during the remained time of the slot, i.e., $T-\tau-\delta\left(\tau+\tau_{h o}\right)$, and the user tries to find a transmission opportunity at the beginning of the next slot.

\footnotetext{
${ }^{1}$ Please note that the main purpose of this study is to evaluate the maximum achievable throughput in a slot by the SU. To this end, as discussed, the transmission rates are assumed the highest possible values, i.e., $C_{0}$ and $C_{1}$, when the SU actually facing free and occupied channels, respectively. So, the nodes $T x H P_{i}$ and $T x L P_{i}$ are considered as terminal nodes.
} 


\section{Model Validation}

In order to compute the average throughput of the SU, the probability of transmitting data from each of the transmitter nodes in Fig. 1 as well as their offered data rates must be derived. Let $\alpha_{x}$ represent the packet arrival rate at node $x$. Considering Fig. 1, the traffic equations of the proposed queuing network can be written as [25]:

$$
\begin{gathered}
\alpha_{H O_{1}}=\lambda+\alpha_{w a i t}, \quad \alpha_{H O_{i}}=q_{i-1} \alpha_{S_{i-1}}, \quad 2 \leq i \leq \delta \\
\alpha_{T x L P_{i}}=\left(P_{i, 1}\left(1-P_{d, i}\right)\right) \alpha_{S_{i}}, \quad 1 \leq i \leq \delta \\
\alpha_{T x H P_{i}}=\left(P_{i, 0}\left(1-P_{f a, i}\right)\right) \alpha_{S_{i}}, \quad 1 \leq i \leq \delta
\end{gathered}
$$

where $q_{i}$ is defined in (2). By simplifying the above equations, the traffics of the nodes are easily computed as,

$$
\begin{gathered}
\alpha_{T x L P_{i}}=\lambda\left(P_{i, 1}\left(1-P_{d, i}\right)\right) \frac{q_{1} q_{2} \ldots q_{i-1}}{1-q_{1} q_{2} \ldots q_{\delta}}, 1 \leq i \leq \delta \\
\alpha_{T x H P_{i}}=\lambda\left(P_{i, 0}\left(1-P_{f a, i}\right)\right) \frac{q_{1} q_{2} \ldots q_{i-1}}{1-q_{1} q_{2} \ldots q_{\delta}}, 1 \leq i \leq \delta
\end{gathered}
$$

The packet is respectively transmitted through the nodes $T x L P_{i}$ and $T x H P_{i}$ with the probabilities of $P_{T x L P_{i}}$ and $P_{T x H P_{i}}$. The transmission probabilities can be easily computed as:

$$
\begin{gathered}
P_{T x L P_{i}}=\frac{\alpha_{T x L P_{i}}}{\alpha_{w a i t}+\sum_{m=1}^{\delta} \alpha_{T x H P_{m}}+\sum_{m=1}^{\delta} \alpha_{T x L P_{m}}} \\
P_{T x H P_{i}}=\frac{\alpha_{T x H P_{i}}}{\alpha_{w a i t}+\sum_{m=1}^{\delta} \alpha_{T x H P_{m}}+\sum_{m=1}^{\delta} \alpha_{T x L P_{m}}}
\end{gathered}
$$

Let $R_{T x L P_{i}}$ and $R_{T x H P_{i}}$ be the average throughputs of the $i$-th stage. Considering (9) and (10), we have,

$$
\begin{aligned}
R_{T x L P_{i}} & =P_{T x L P_{i}} \frac{R T_{i}}{T} C_{1} \\
R_{T x H P_{i}} & =P_{T x H P_{i}} \frac{R T_{i}}{T} C_{0}
\end{aligned}
$$

where $R T_{i}$ is defined and given in (1). Then, the average SU throughput can be calculated as:

$$
R=\sum_{i=1}^{\delta} R_{T x L P_{i}}+\sum_{i=1}^{\delta} R_{T x H P_{i}}
$$

Substituting (3), (11), and (12) in (13) and simplifying the result, the SU's throughput is obtained in (14), where $q_{0} \triangleq 1$. Since the proposed model will be extended to the multiple SUs case, to validate the model and to verify the approach, we have also directly computed the system throughput for the single SU case in Appendix A, and obtained exactly the same result. The result will be further verified by simulation in numerical result section.

\section{Modified P-Persistent Access Scheme}

In this section, we extend the considered sequential channel sensing approach by modifying the queue network proposed in Fig. 1 to include the multiuser CRN. In order to provide multiple access among the SUs, a modified version of the conventional p-persistent multiple access protocol is utilized in which each SU senses each channel with the probability $p$ and skips the sensing process with the probability $(1-p)$. The channel sensing probability, $p$, provides a degree of freedom to optimize the performance metrics, namely, the throughput of SUs. Fig. 2 illustrates the channel sensing-access policy of the proposed modified p-persistent access protocol (MPPA) used by each SU. Since at each stage $i$ some requests may not be routed to the node $S_{i}$, with the probability of $(1-p)$, as discussed above, we add nodes $S Y N_{i}$ in Fig. 2, in order to synchronize the requests. More specifically, the requests that enter standby mode (at node $S Y N_{i}$ ) wait for $\tau$ time units (sensing period). Then, they are directed to the node $H_{i+1}$. With the help of the synchronizer nodes, all requests will enter the $i$-th sensing node at the same time. As a result, the $i$-th channel will be busy at the beginning of the corresponding sensing interval if and only if the $i$-th PU is present. At first, all requests enter the node $H O_{1}$. Then, they are routed to the first sensing node, $S_{1}$, with the probability of $p$, or to the second handover node, $\mathrm{HO}_{2}$, after spending $\tau$ time units in the first synchronizer node $S Y N_{1}$, with the probability of $(1-p)$. Based on the sensing results, packets in the node $S_{1}$ are directed to the first transmitter node or the second handover node with the probabilities shown in Fig. 2. The requests follow the same processes until a transmission opportunity is found or the handover limitation, discussed in (3), is reached. These requests must wait for the rest of the current time slot. Also note that the $i$-th stage of the MPPA protocol comprise of the nodes $H O_{i}, S Y N_{i}, S_{i}, T x L P_{i}$, and $T x H P_{i}$. The other parameters in Fig. 2 are defined as in Fig. 1.

To evaluate the performance of the MPPA scheme, we exploit the same steps as taken in section II-C. Considering Fig. 2 and superscript $[k]$ for the $k$-th $\mathrm{SU}$, the departure rate for user $\mathrm{k}$ is computed as,

$$
\begin{gathered}
\alpha_{H O_{i}}^{[k]}=c_{i-1} \alpha_{H O_{i-1}}^{[k]}, \quad 2 \leq i \leq \delta \\
\alpha_{w_{a i t}^{[k]}}^{[k]} q_{\delta} \alpha_{S_{\delta}}^{[k]}+(1-p) \alpha_{H O_{\delta}}^{[k]}=c_{\delta} \alpha_{H O_{\delta}}^{[k]}
\end{gathered}
$$

where $c_{i} \triangleq 1-p+p q_{i}$ indicates the probability of handover event from the stage $i$ to the stage $i+1$. By defining $c_{0} \triangleq 1$ and taking the same procedures as in the section II-C, we have:

$$
\alpha_{H O_{i}}^{[k]}=\lambda^{[k]} \frac{c_{1} c_{2} \cdots c_{i-1}}{1-c_{1} c_{2} \cdots c_{\delta}}, 1 \leqslant i \leqslant \delta
$$

$$
\alpha_{T x L P_{i}}^{[k]}=\left(P_{i, 1}\left(1-P_{d, i}\right)\right) p \lambda^{[k]} \frac{c_{1} c_{2} \cdots c_{i-1}}{1-c_{1} c_{2} \cdots c_{\delta}}, 1 \leq i \leq \delta
$$

$\alpha_{T x H P_{i}}^{[k]}=\left(P_{i, 0}\left(1-P_{f a, i}\right)\right) p \lambda^{[k]} \frac{c_{1} c_{2} \cdots c_{i-1}}{1-c_{1} c_{2} \cdots c_{\delta}}, 1 \leq i \leq \delta$

Let $P_{T x L P_{i}}^{([k]}$ and $P_{T x H P_{i}}^{[k]}$ denote the transmission probabilities of the $k$-th SU from the nodes $T x L P_{i}$ and $T x H P_{i}$, 


$$
R=\sum_{m=1}^{\delta} q_{0} q_{1} \cdots q_{m-1}\left(C_{1} P_{m, 1}\left(1-P_{d, m}\right)+C_{0} P_{m, 0}\left(1-P_{f a, m}\right)\right)\left(1-\frac{\tau+(m-1)\left(\tau+\tau_{h o}\right)}{T}\right)
$$

$$
R^{[k]}=\frac{1}{T} \sum_{i=1}^{\delta} c_{0} c_{1} \cdots c_{i-1}\left(P_{i, 1}\left(1-P_{d, i}\right) C_{1}+P_{i, 0}\left(1-P_{f a, i}\right) C_{0}\right) p\left(1-c_{0} c_{1} \cdots c_{i-1}\left(1-c_{i}\right)\right)^{N_{s}-1} R T_{i}
$$

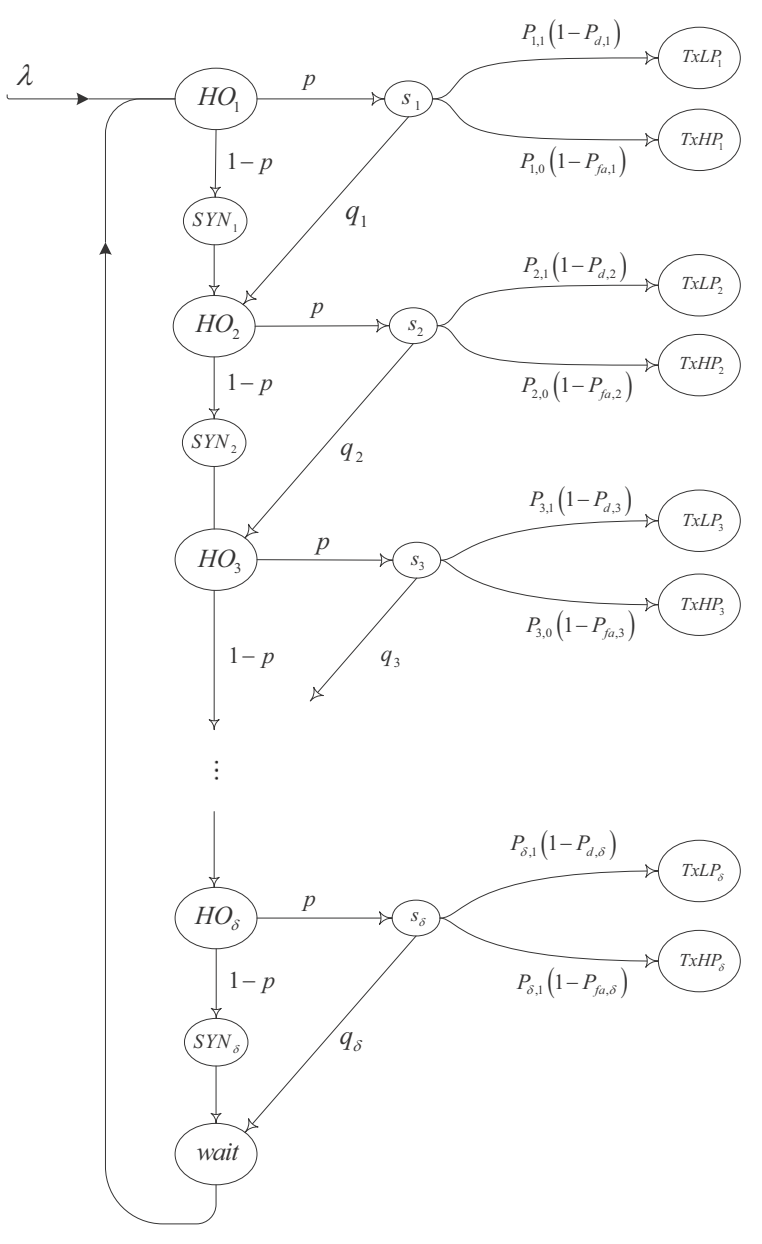

Fig. 2. The Markov process of modified p-persistent access (MPPA) scheme for each secondary user's sensing-transmission process.

respectively. Similar to (9)-(10), they can be computed:

$$
\begin{aligned}
P_{T x L P_{i}}^{[k]} & =\frac{\alpha_{T x L P_{i}}^{[k]}}{\alpha_{w a i t}^{[k]}+\sum_{m=1}^{\delta} \alpha_{T x H P_{m}}^{[k]}+\sum_{m=1}^{\delta} \alpha_{T x L P_{m}}^{[k]}} \\
P_{T x H P_{i}}^{[k]} & =\frac{\alpha_{T x H P_{i}}^{[k]}}{\alpha_{w a i t}^{[k]}+\sum_{m=1}^{\delta} \alpha_{T x H P_{m}}^{[k]}+\sum_{m=1}^{\delta} \alpha_{T x L P_{m}}^{[k]}}
\end{aligned}
$$

To compute the average throughput, it must be noted that there will be a successful data transmission from the $i$-th stage of the $k$-th SU if among all SUs, only the $k$-th SU tries to transmit on this channel either from the node $\mathrm{TxHP}_{i}$ or from the node $T x L P_{i}$. Thus, we have,

$$
\begin{aligned}
\mathcal{P}_{T x L P, i, k} & =\prod_{\substack{m \neq 1 \\
m \neq k}}^{N_{S}} P_{T x L P_{i}}^{[k]}\left(1-P_{T x L P_{i}}^{[m]}-P_{T x H P_{i}}^{[m]}\right) \\
\mathcal{P}_{T x H P, i, k} & =\prod_{\substack{m=1 \\
m \neq k}}^{N_{S}} P_{T x H P_{i}}^{[k]}\left(1-P_{T x L P_{i}}^{[m]}-P_{T x H P_{i}}^{[m]}\right)
\end{aligned}
$$

where $\mathcal{P}_{T x L P, i, k}$ and $\mathcal{P}_{T x H P, i, k}$ represent the successful transmission of each user $k$ from the nodes $T x L P_{i}$ and $T x H P_{i}$ without collision among the SUs, respectively. Finally from (1), (22), and (23), the average throughput of each user $k$ can be easily calculated as:

$$
R^{[k]}=\frac{1}{T} \sum_{i=1}^{\delta} \mathcal{P}_{T x L P, i, k} R T_{i} C_{1}+\mathcal{P}_{T x H P, i, k} R T_{i} C_{0}
$$

In order to maximize the SUs' performance, the exploited p-persistent protocol must be optimized. It can be optimized through modifying the protocol [29] or efficient designing of its parameters including the parameter $p$ [30], [31]. However, those optimization approaches cannot be directly and easily applied to our proposed models. In the following, two approaches are proposed to maximize the SUs throughputs.

First approach: Here, we aim to find the optimal value of $p$ used in the p-persistent protocol. To this end, we first simplify the equation (24), describing the average achievable throughput. By substituting (16)-(19) into (20)-(24), and after some algebraic manipulations, the average throughput of each $\mathrm{SU} k$ is simplified to (25).

In order to achieve the best performance, the optimal value of $p$ must be determined through the following optimization problem:

$$
p^{*}=\underset{p}{\arg \max } R^{[k]}
$$

The objective function in (26) is generally non-convex, making it difficult to efficiently be solved for the global optimum. In most cases, suboptimal solutions or heuristics approaches have to be used. In this paper, we exploit wellknown steepest descent (SD) and exhaustive search schemes to respectively find the local and global maximum of $R^{[k]}$.

Second approach: It is worth noting that the optimum value of $p$ derived through conventional analytical optimization procedures directly depends on the models adopted for the channel, the sensing errors, and the PUs behavior. Despite the increased analytical complexity introduced by using more precise modeling, if any changes occur in the parameters describing the models, all the analyses and the evaluations have to be repeated in order to find the optimum value of $p$. In order to deal with these issues appropriately, as a second 
solution, we propose a more practical and simpler protocol for the SUs to enhance their average throughputs through properly tuning the value of $p$. Suppose that each SU periodically calculates its average throughput by inspecting the packets and their acknowledgments (ACKs) at the secondary transmitter for a period of time equal to $T_{e p}$ (estimation period) [32]. $T_{e p}$, as a design parameter, depends on the PUs activities and the link behavior. Then, computing the average throughput in two consecutive estimation intervals, the SU can modify the value of $p$. We propose that in the $i$-th interval, $p$ is adaptively determined by the following formula:

$$
p_{i+1}=\min \left(\max \left(p_{i}+\Delta p, 0\right), 1\right)
$$

where for instance

$$
\Delta p=\left\{\begin{array}{cc}
0.1 & T h r_{i} \geq T h r_{i-1} \\
-0.1 & T h r_{i}<T h r_{i-1}
\end{array}\right.
$$

and $T h r_{i}$ denotes the average throughput calculated in the $i$-th interval, and $p_{i}$ represents the value of $p$ adopted for the $i$-th interval. It is worth noting that the function $p_{i}=$ $\min (\max (\cdot, 0), 1)$ is used to limit the value of $p$ within the range of $[0,1]$. Following this protocol, if the network is not highly congested, the SU raises the value $p_{i+1}$, and hence increases the probability of sensing and then opportunistically data transmission as well. On the other hand, if more SUs struggle for accessing the network, each SU reduces its $p_{i+1}$ to decrease the collision level. As a corollary, a higher average throughput can be achieved. Please note that the adaptive configuration scheme proposed requires no prior knowledge about the link behavior, such as the presence or absence probabilities of the PUs. Moreover, it is capable to effectively follow the variations in the link and to keep the average throughput at a high level, as will be demonstrated in the numerical results section.

Besides the average throughput of the SUs, the energy consumed in the handover procedure is another remarkable performance metric, especially for energy-aware protocols, and can be effectively used to assess different schemes. Hence, in the following, the average number of required handovers, or equivalently the average consumed energy, is derived. Note that the channel $i$ will be chosen for the transmission if and only if (a) all $(i-1)$ previous channels are either not sensed due to the exploited p-persistent MAC protocol or sensed busy, (b) the channel $i$ is sensed idle. So the $k$-th SU requires $(i-1)$ times handovers with the probability of $c_{1} c_{2} \ldots c_{i-1}\left(1-c_{i}\right)$, where $c_{i}$, the transition probability from the stage $i$ to the stage $i+1$, is given in (16). The average number of handovers, denoted by $N_{H O}$, is easily computed as,

$$
N_{H O}=\sum_{i=1}^{\delta}(i-1)\left(1-c_{i}\right) \prod_{j=1}^{i-1} c_{j}+\delta \prod_{j=1}^{\delta} c_{j}
$$

where the last term is corresponding to the possibility of no transmission in any of $\delta$ transmission stages.

\section{P-Persistent Random Access Scheme}

The major disadvantage associated with the MPPA algorithm is that a high number of SUs ( $p N_{p}$ SUs) intend to sense the first channel through the node $S_{1}$. Similarly, the $c_{1} N_{p}$

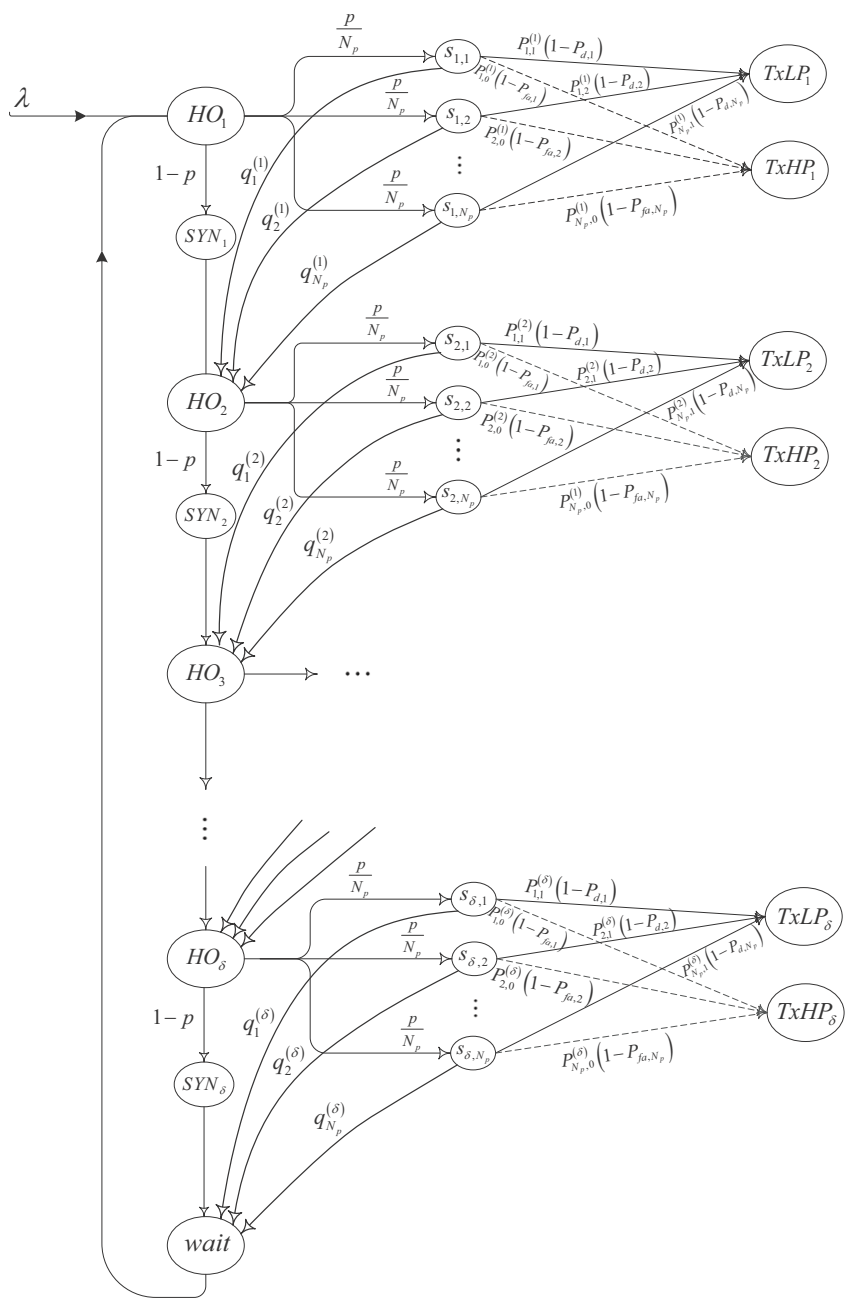

Fig. 3. The Markov process of p-persistent random access (PPRA) scheme for each secondary user's sensing-transmission process.

SUs' requests enter the node $S_{2}$, and so forth. While this structure facilitates the network modeling and the performance evaluation, it imposes a high level of contention among the SUs to exploit the spectrum resources in each stage and consequently degrades the performance regarding the average SUs' throughput. In order to mitigate the aforementioned problem, we consider the p-persistent random access (PPRA) scheme, which equally distributes the load of the SUs within all channels, and hereby decreases the contention level and raises the throughput of each SU.

Fig. 3 demonstrates the sensing-transmission stages of the proposed PPRA method. As it shows, at the $i$-th stages, a request is routed to the sensing nodes, $S_{i, 1}, S_{i, 2}, \ldots, S_{i, N_{p}}$, corresponding to the channels $1,2, \ldots, N_{p}$, respectively, with the identical probabilities of $\frac{p}{N_{s}}$, after running a p-persistent protocol as described in the previous section. Then, the request will be directed to the corresponding transmission nodes, i.e., $T x L P_{i}$ or $T x H P_{i}$, or the next handover node, i.e., $H O_{i+1}$. Regardless of the exploited load balancing technique, other processes and parameters are the same as the MPPA scheme. 


\section{A. Performance Analysis}

In the PPRA model, a channel, which is sensed busy by an SU, can be occupied either by the PU or the other SUs; because it may have been detected as a transmission opportunity by another SU at the previous stages. Therefore, in this model, the impact of other SUs' transmissions must be considered on the channels occupation probabilities.

Lemma 1: The occupation probability of $i$-th channel at the beginning of $m$-th stage, denoted by $P_{i, 1}^{(m)}$, is obtained as,

$$
P_{i, 1}^{(m)}=P_{i, 1}^{(m-1)}+P_{i, 0} P_{f a, i} L^{(1)}+L^{(2)}+\cdots+L^{(m-2)} U_{i}^{(m-1)}
$$

where $L^{(m)}$ represents the number of the SUs sensing the $i$ th channel at stage $m$, and $U_{i}^{(m)}$ denotes the probability of transmission on the $i$-th channel at the stage $m$ by at least one SU conditioned on the absence of the corresponding PU. $P_{f a, i}$ is the false alarm probability of the $i$-th channel.

Proof: The proof is given in Appendix B.

Proposition: The $i$-th channel is sensed busy at the $m$-th stage with the following probability:

$$
q_{i}^{(m)}=P_{i, 0}^{(m)} P_{f a, i}+P_{i, 1}^{(m)} P_{d, i}, \quad 1 \leqslant i \leqslant N_{p}
$$

From Fig. 3, for the $k$-th SU $\alpha_{H O_{i}}^{[k]}$ is obtained in (32), where $q_{j}^{(i)}$ is defined in (31). From another perspective, the $k$-th SU may perform a handover and transit from the $i$-th stage to the $(i+1)$-th stage. If we denote this probability by $c_{i}^{[k]}$, we have,

$$
\alpha_{H O_{i+1}}^{[k]}=c_{i}^{[k]} \alpha_{H O_{i}}^{[k]}, \quad 1 \leq i \leq \delta
$$

Taking (32) and (33) into account, $c_{i}^{[k]}$ for the PPRA protocol is calculated as,

$$
c_{i}{ }^{[k]}=1-p+p \frac{q_{1}^{(i)}+q_{2}^{(i)}+\cdots+q_{N_{p}}^{(i)}}{N_{p}}
$$

where $c_{0}^{[k]} \triangleq 1$. Since the derived probability does not depend on the superscript $[k]$, we omit the superscript and simply represent this parameter by $c_{i}$, which is the transition probability between two consecutive stages, similar to the definition given in (15)-(19) for the MPPA scheme. By the same steps taken in section III, we have, $\alpha_{\text {wait }}^{[k]}=c_{\delta} \alpha_{H O_{\delta}}^{[k]}$, and,

$$
\alpha_{H O_{i}}^{[k]}=\lambda^{[k]} \frac{c_{1} c_{2} \cdots c_{j-1}}{1-c_{1} c_{2} \cdots c_{\delta}}, \quad 1 \leqslant i \leqslant \delta
$$

To evaluate the departure rates of the transmitter nodes of the $i$-th stage of the $k$-th SU, i.e., $\alpha_{T x L P_{i}}^{[k]}$ and $\alpha_{T x H P_{i}}^{[k]}$, first note that $\alpha_{S_{i, j}}^{[k]}=\frac{p}{N_{p}} \alpha_{H O_{i}}^{[k]}$ for $1 \leq i \leq \delta$ and $1 \leq j \leq N_{p}$. Then, $\alpha_{T x L P_{i}}^{[k]}$ is simply calculated in (36), where $P_{d, j}$ is the detection probability of channel $j$, and $P_{j, 1}^{(i)}$ is defined in (30). If we define $d_{1, i} \triangleq \frac{1}{N_{p}} \sum_{j=1}^{N_{p}} P_{j, 1}^{(i)}\left(1-P_{d, j}\right)$, (36) is simplified to:

$$
\alpha_{T x L P_{i}}^{[k]}=\lambda^{[k]} p d_{1, i} \frac{c_{1} c_{2} \cdots c_{i-1}}{1-c_{1} c_{2} \cdots c_{\delta}}, \quad 1 \leqslant i \leqslant \delta
$$

Similarly, for the node $\operatorname{TxH}_{i}$ is derived in (38), where $d_{0, i}=\frac{1}{N_{p}} \sum_{j=1}^{N_{p}} P_{j, 0}^{(i)}\left(1-P_{f a, j}\right)$.

Let $\alpha_{T x L P_{i} \mid S_{i, j}}^{[k]}$ and $\alpha_{T x H P_{i} \mid S_{i, j}}^{[k]}$ respectively represent the departure rates of the nodes $T x L P_{i}$ and $T x H P_{i}$ initiated from the node $S_{i, j}$ (please see Fig. 3). From Fig. 3, we have:

$$
\begin{gathered}
\alpha_{T x L P_{i} \mid S_{i, j}}^{[k]}=P_{j, 1}^{(i)}\left(1-P_{d, j}\right) \alpha_{S_{i, j}}^{[k]}=\frac{p}{N_{p}} P_{j, 1}^{(i)}\left(1-P_{d, j}\right) \alpha_{H O_{i}}^{[k]} \\
\alpha_{T x H P_{i} \mid S_{i, j}}^{[k]}=P_{j, 0}^{(i)}\left(1-P_{f a, j}\right) \alpha_{S_{i, j}}^{[k]}
\end{gathered}
$$

Further, assume that $P_{T x L P_{i}, S_{i, j}}^{[k]}$ and $P_{T x H P_{i}, S_{i, j}}^{[k]}$ denote the probabilities of transmission from the node $T x L P_{i}$ and $T x H P_{i}$ on the $j$-th channel, respectively, i.e., the request is directed from the node $S_{i, j}$ to the node $T x L P_{i}$ or $T x H P_{i}$. Then, $P_{T x L P_{i}, S_{i, j}}^{[k]}$ is derived in (41), and similarly,

$$
P_{T x H P_{i}, S_{i, j}}^{[k]}=\frac{\alpha_{T x H P_{i} \mid S_{i, j}}^{[k]}}{\alpha_{w a i t}^{[k]}+\sum_{i=1}^{\delta} \alpha_{T x H P_{i}}^{[k]}+\sum_{i=1}^{\delta} \alpha_{T x L P_{i}}^{[k]}}
$$

where $\alpha_{T x L P_{i}}^{[k]}$ and $\alpha_{T x H P_{i_{i}}}^{[k]}$ are derived in (37) and (38), respectively. The $k$-th SU will be able to successfully transmit data from the $i$-th stage with the probability of:

$$
\begin{aligned}
& \mathcal{P}_{T x L P, i, k}=\sum_{j=1}^{N_{p}} \prod_{\substack{m=1 \\
m \neq k}}^{N_{S}} P_{T x L P_{i}, S_{i, j}}^{[k]} \chi_{i, j}^{[m]} \\
& \mathcal{P}_{T x H P, i, k}=\sum_{j=1}^{N_{p}} \prod_{\substack{m=1 \\
m \neq k}}^{N_{S}} P_{T x H P_{i}, S_{i, j}}^{[k]} \chi_{i, j}^{[m]} \\
& \chi_{i, j}^{[m]}=1-P_{T x L P_{i}, S_{i, j}}^{[m]}-P_{T x H P_{i}, S_{i, j}}^{[m]}
\end{aligned}
$$

and finally, the average throughput of each SU $k$ is computed by substituting (43)-(44) into (24). Similar to the MPPA model, the average throughput achieved in the PPRA model can be locally, globally, or adaptively optimized by applying the same approaches described for the MPPA scheme.

By following the same procedures as in section III, the average number of required handovers for the PPRA scheme can be obtained by substituting the corresponding $c_{i}$, provided in (34), into (29).

\section{NumericAl Results}

In this section, the performances of the proposed schemes are evaluated and compared by the analytical results and simulations considering the effects of different parameters introduced throughout the paper. The values of parameters used for the numerical evaluation are given in Table I. The energy detector (ED), as the most prevalent channel sensor for the CRNs in the literature [33], is exploited to simulate the sensing nodes. The detailed structure and performance of the ED can be found in [34]. The value of sampling frequency is adopted from [26], and the minimum allowable value of detection probability, $P_{d}^{\min }$, the maximum admissible false alarm probability, $P_{f a}^{\max }$, and the time slot duration, $T$, are chosen according to IEEE 802.22 standard [28] and [35]. 


$$
\alpha_{H O_{i}}^{[k]}=(1-p) \alpha_{H O_{i-1}}^{[k]}+\frac{p}{N_{p}}\left(q_{1}^{(i-1)}+q_{2}^{(i-1)}+\cdots+q_{N_{P}}^{(i-1)}\right) \alpha_{H O_{i-1}}^{[k]}=\left(1-p+p \frac{\sum_{j=1}^{N_{p}} q_{j}^{(i-1)}}{N_{p}}\right) \alpha_{H O_{i-1}}^{[k]}, \quad 2 \leqslant i \leqslant N_{p}
$$

$$
\alpha_{T x L P_{i}}^{[k]}=P_{1,1}^{(i)}\left(1-P_{d, 1}\right) \alpha_{S_{i, 1}}^{[k]}+\cdots+P_{N_{p}, 1}^{(i)}\left(1-P_{d, N_{p}}\right) \alpha_{S_{i, N_{p}}}^{[k]}=\left(\frac{\sum_{j=1}^{N_{p}} P_{j, 1}^{(i)}\left(1-P_{d, j}\right)}{N_{p}}\right) p \alpha_{H O_{i}}^{[k]}, \quad 1 \leqslant i \leqslant \delta
$$

$$
\alpha_{T x H P_{i}}^{[k]}=\sum_{j=1}^{N_{p}} P_{j, 0}^{(i)}\left(1-P_{f a, j}\right) \alpha_{S_{i, j}}^{[k]}=\left(\frac{\sum_{j=1}^{N_{p}} P_{j, 0}^{(i)}\left(1-P_{f a, j}\right)}{N_{p}}\right) p \alpha_{H O_{i}}^{[k]}=\lambda^{[k]} p d_{0, i} \frac{c_{1} c_{2} \cdots c_{i-1}}{1-c_{1} c_{2} \cdots c_{\delta}}, \quad 1 \leqslant i \leqslant \delta
$$

$$
P_{T x L P_{i}, S_{i, j}}^{[k]}=\frac{\alpha_{T x L P_{i} \mid S_{i, j}}^{[k]}}{\alpha_{w a i t}^{[k]}+\sum_{i=1}^{\delta} \sum_{j=1}^{N_{p}} \alpha_{T x H P_{i} \mid S_{i, j}}^{[k]}+\sum_{i=1}^{\delta} \sum_{j=1}^{N_{p}} \alpha_{T x L P_{i} \mid S_{i, j}}^{[k]}}=\frac{\alpha_{T x L P_{i} \mid S_{i, j}}^{[k]}}{\alpha_{w a i t}^{[k]}+\sum_{i=1}^{\delta} \alpha_{T x H P_{i}}^{[k]}+\sum_{i=1}^{\delta} \alpha_{T x L P_{i}}^{[k]}}
$$

TABLE I

SiMULATION PARAMETERS

\begin{tabular}{ccc}
\hline \hline Parameter & Description & Value \\
\hline$P_{d}^{\min }$ & Minimum allowable detection probability & 0.9 \\
$P_{f a}^{\max }$ & Maximum allowable false alarm probability & 0.1 \\
$f_{s}$ & Receiver sampling frequency & $6 \mathrm{MHz}$ \\
$T$ & Time-slot duration & $10 \mathrm{~ms}$ \\
$\tau_{h o}$ & Required time for handover & $0.01 \mathrm{~ms}$ \\
$\gamma_{s}$ & Received SNR due to the SU signal & $5 \mathrm{~dB}$ \\
$\gamma_{p}$ & Received SNR due to the PU signal & $5 \mathrm{~dB}$ \\
\hline \hline
\end{tabular}

In simulation evaluations, the average throughput has been computed after simulating each scenario for 100 time slots. In Figs. 4 and 5, a CRN with one SU, and in Figs. 6-11, a CRN with multiple SUs case are considered.

Fig. 4 shows the average throughput ${ }^{2}$ of the single SU using sequential channel sensing scheme versus normalized sensing time (i.e., $\tau / T$ ) based on both analytical and simulation results. From this Fig., it can be realized that the simulation results well coincide the analytical results, which further validates our analytical derivations. In general, as the sensing time increases, the detector senses the channels more accurately and finds more transmission opportunities. However, by increasing the sensing time, less time remains for the transmission. Hence, there exists a tradeoff between the average SU's throughput and its detection accuracy. As it can be seen in Fig. 4, first the SU throughput increases by $\tau$ (due to more accurate sensing); then after an optimum point where $P_{d}$ and $P_{f a}$ are in an acceptable level, the throughput starts decreasing due to the reduction of the time left for the transmission. From Fig. 4, there exists an optimum value for the spectrum sensing time.

${ }^{2}$ Please note that the average throughput has been normalized to $C_{0}$.

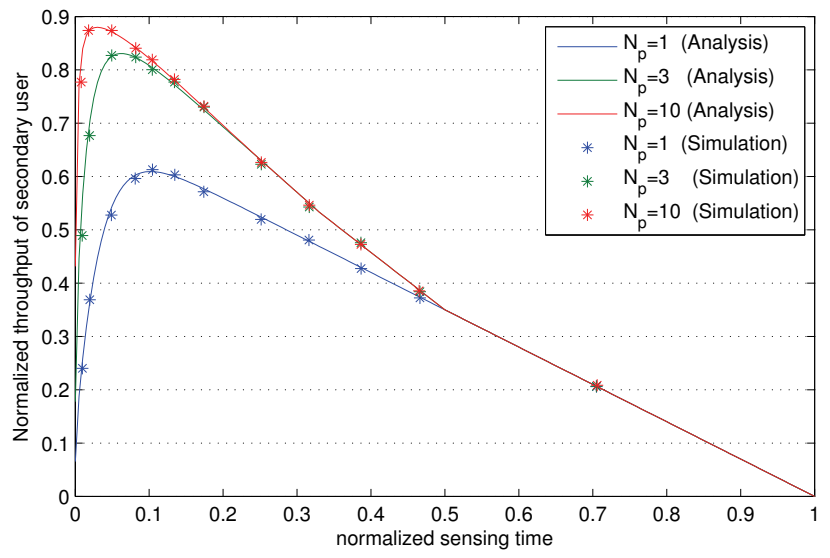

Fig. 4. Secondary user normalized throughput versus normalized sensing time for a CRN with single secondary user.

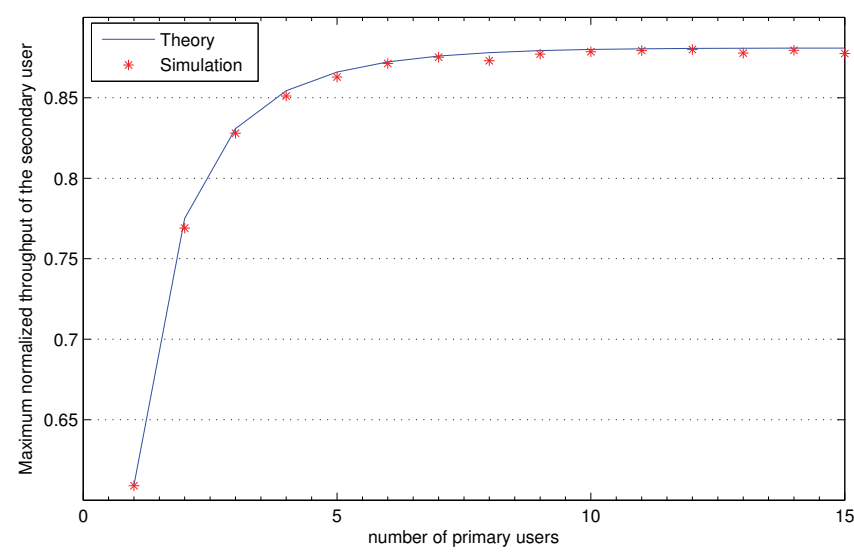

Fig. 5. The maximum normalized throughput versus the number of primary users for a CRN with one secondary user. 
Fig. 5 further verifies our analysis and represents the plot of the maximum achievable throughput, corresponding to the optimum value of the spectrum sensing time, versus the number of PUs. Main points highlighted in Fig. 5 are twofold. First, as the number of primary channels increases, the SU throughput increases as well, but in a saturating manner. This is due to the fact that, though the average number of obtained transmission opportunities increases by the number of primary channels, but the average time left for the transmission reduces. Second, this figure clearly demonstrates the importance and efficiency of having multiple handovers. Interestingly, the improvement in the SU's maximum throughput when using multiple handovers is about $44.5 \%$ compared to the case of $N_{p}=1$, with no handover capability, for the example considered.

Fig. 6 illustrates the plot of the average throughput of an SU versus the normalized sensing time for the MPPA scheme considering a primary network with 10 PUs and a secondary network with 5 SUs. Increasing the channel sensing time, $\tau$, affects the SUs' average throughput as discussed in Fig. 4. Moreover, increasing the channel sensing probability raises the contention level among the SUs and consequently reduces their average throughputs.

The energy efficiency of the PPRA scheme compared to the MPPA strategy is demonstrated through Fig. 7, which shows the plot of the SUs' average throughput versus the average number of handovers assuming that $\tau / T=0.24$. Due to the load distributing nature of the PPRA scheme, this protocol achieves remarkably higher average throughput for a certain number of handovers. Specifically, for $N_{H O}=3.72$, the throughput achieved by the PPRA is about 3.5 times more than the one achieved by the MPPA scheme.

Figs. 8 and 9 further verify the advantages of the PPRA algorithm compared to the MPPA schemes. These figures respectively compare the average throughput and the collision probability of an SU obtained by the proposed MPPA and the PPRA schemes for the case of $N_{p}=8, N_{s}=5$, and $\tau / T=0.24$. As can be realized, the average throughput obtained by the MPPA scheme is lower than the one achieved by the PPRA scheme. In both schemes, the SUs sense the channels with higher probability as the channel sensing probability, $p$, increases. Although the increase of $p$ can raise the chance of finding a transmission opportunity, it increases the average level of contention among the SUs. Therefore, there is a tradeoff on the value of $p$, and it can be optimally designed in order to reach the maximum average throughput. For the example considered, the maximum throughput achieved by the PPRA scheme is about 3.63 times more than that of the MPPA scheme. Also, as less requests compete for accessing the same channel in the PPRA scheme, the breaking point of the PPRA's average throughput is greater than the MPPA protocol. In addition, as Fig. 9 shows, less collision is imposed by the PPRA scheme compared to the MPPA due to the exploited load balancing method.

To more clearly illustrate the performance enhancement due to optimal p-persistent policies, Table II compares the average throughput for four different cases: 1) a typical value of $p$, i.e., $p=0.8,2)$ global optimum value of $p$, which is obtained through the exhaustive search, 3) a local optimum value of $p$, which is obtained through the SD algorithm for

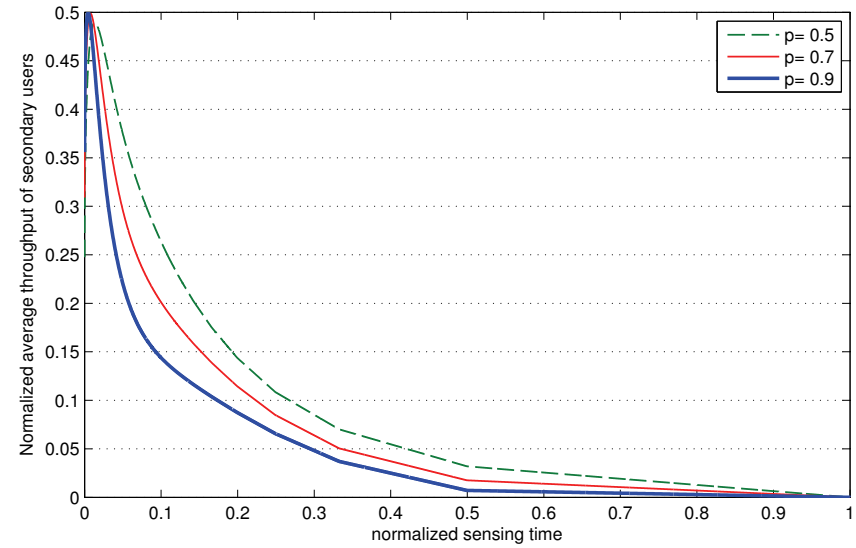

Fig. 6. Average secondary user normalized throughput versus normalized sensing time.

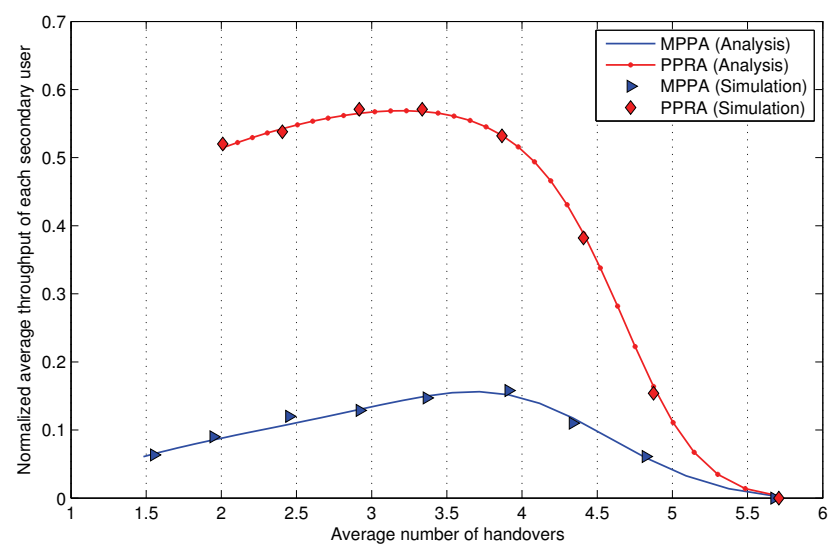

Fig. 7. Average secondary user normalized throughput versus average number of handovers.

three different initial values, and 4) a proper value of $p$, which is obtained through adaptive protocol proposed. As expected, adopting the optimal and suboptimal p-persistent policies increase the average throughput. The exhaustive search grants the maximum throughput at the cost of massive computational burden. The SD algorithm well approximates the optimal value of the throughput for three different examples considered for its initial values. Albeit this algorithm cannot guarantee the global optimum value of $p$, its much lower computational burden is a key advantage. Finally, for the adaptive protocol proposed, the SUs throughput remains at an acceptable level while no information about other SUs (even $N_{s}$ ) is required. Intuitively, selecting a method to find a proper value highly depends on the information available and the tradeoff between the computational cost affordable and the optimality of the solution desirable.

The SUs' average collision probability at each stage are depicted in Fig. 10 for both MPPA and PPRA schemes. In the first stages, the transmitter nodes face more collisions, and the probability of collision reduces as the requests intend to transmit in the higher stages. Again, the MPPA protocol leads to collision among the SUs with higher probability than the PPRA scheme.

Finally, we investigate the performance of a simple improvement of the PPRA scheme, called improved-PPRA. In 
TABLE II

IMPACT OF OPTIMAL P-PERSISTENT PROTOCOL ON THE AVERAGE THROUGHPUT OF THE SU $k$.

\begin{tabular}{c|c|cc|cc|cc|cc}
\hline \hline & {$\left[N_{s}=1, N_{p}=5\right]$} & \multicolumn{2}{|c|}{$\left[N_{s}=3, N_{p}=5\right]$} & {$\left[N_{s}=5, N_{p}=5\right]$} & {$\left[N_{s}=5, N_{p}=8\right]$} \\
\cline { 2 - 11 } & MPPA & PPRA & MPPA & PPRA & MPPA & PPRA & MPPA & PPRA \\
\hline \multicolumn{2}{c|}{$p=0.8$} & 0.7072 & 0.7113 & 0.3315 & 0.5824 & 0.116 & 0.4602 & 0.0737 & 0.555 \\
\hline \multirow{2}{*}{ Exhaustive search } & 0.7471 & 0.7584 & 0.3596 & 0.5945 & 0.1534 & 0.5153 & 0.1848 & 0.5689 \\
\hline \multirow{3}{*}{ The SD algorithm } & $p_{0}=0.1$ & 0.747 & 0.7562 & 0.3594 & 0.5939 & 0.1531 & 0.5152 & 0.1848 & 0.5684 \\
\cline { 2 - 10 } & $p_{0}=0.5$ & 0.7471 & 0.7556 & 0.3596 & 0.5941 & 0.1533 & 0.5151 & 0.1847 & 0.5682 \\
\cline { 2 - 9 } & $p_{0}=0.9$ & 0.7471 & 0.7551 & 0.3595 & 0.5943 & 0.1532 & 0.5152 & 0.1844 & 0.5689 \\
\hline \multicolumn{2}{l|}{ Adaptive protocol } & 0.7471 & 0.7493 & 0.3579 & 0.591 & 0.1487 & 0.5086 & 0.1829 & 0.5675 \\
\hline \hline
\end{tabular}

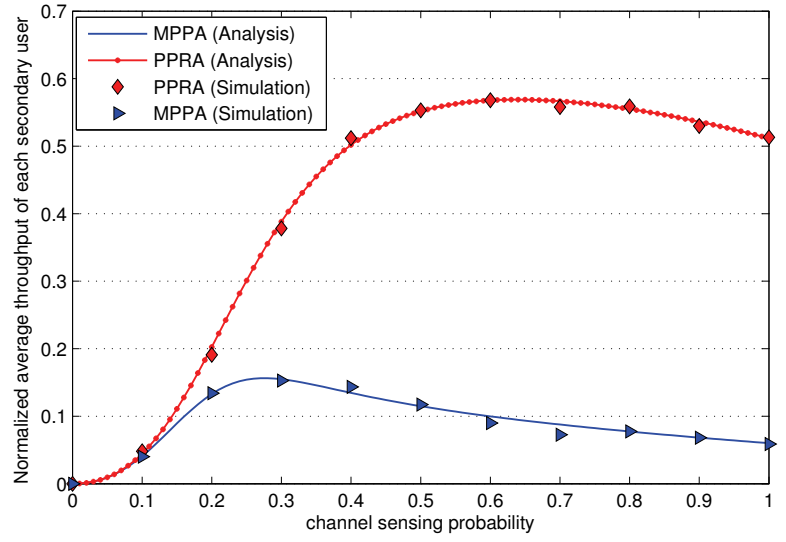

Fig. 8. Maximum normalized throughput of each secondary user versus the channel sensing probability.

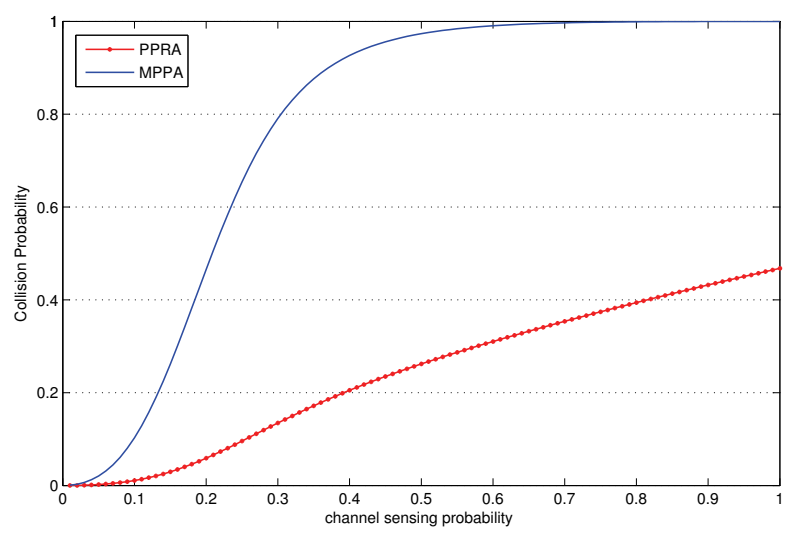

Fig. 9. Average collision probability of requests versus the channel sensing probability.

contrast to the PPRA scheme, in which in each random access stage, a sensing channel is selected uniformly from all the $N_{p}$ channels, in the improved scheme, once an SU senses a PU channel occupied, the SU avoid sensing this channel in later random access stages. Fig. 11 compares the the average throughputs of the PPRA scheme versus the improved PPRA scheme. As can be observed, the average throughput is slightly raised. The results presented in Fig. 11 are based on simulation, since the analytical performance evaluation of the improved PPRA protocol is highly complicated. From the results observed, the performance of the proposed PPRA scheme, analytically formulated in (30)-(44), can be considered as a tight lower bound of the improved PPRA protocol.

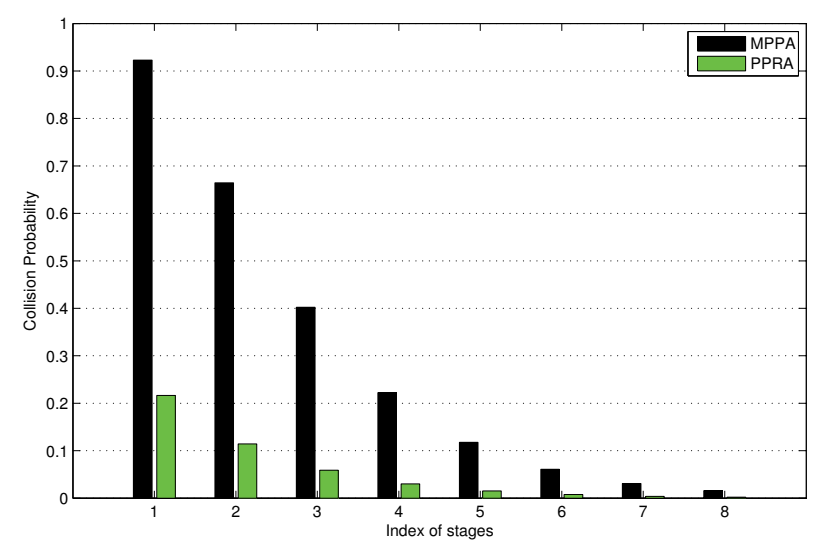

Fig. 10. Average collision probability of requests in various stages.

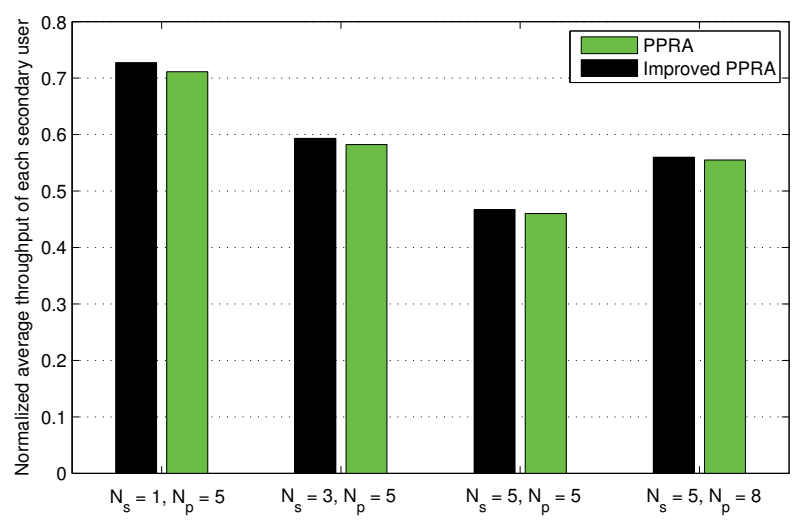

Fig. 11. Comparison between the average achievable throughputs of the PPRA and improved-PPRA schemes for $p=0.8$.

\section{CONCLUSION}

Designing, modeling, and systematic performance evaluating of sequential channel sensing schemes in a multichannel cognitive radio networks (CRNs) have been investigated. First, finite state Markovian process-based structure has been exploited to effectively model the behavior of a single secondary user (SU) in the CRN. This model has been validated by analytical analysis and simulations, and then extended to a multiuser CRN. Modified p-persistent access (MPPA) has been introduced, and its performance in terms of the average SUs' throughput and the average number of handovers has been evaluated. In order to appropriately mitigate the problem associated with the MPPA scheme, a distributed sensingaccess policy, called p-persistent random access (PPRA), has been proposed, which statistically distributes the SUs' 
load among all channels. Consequently, as discussed in the paper, this scheme provides lower contention among SUs for accessing same channels and offers higher average throughput, as demonstrated through exhaustive numerical performance evaluations.

\section{APPENDiX A}

Lemma 1: The average achievable throughput can be calculated as,

$$
R=\sum_{m=1}^{\delta} q_{0} q_{1} \cdots q_{m-1} \kappa_{m}\left(1-\frac{\tau+(m-1)\left(\tau+\tau_{h o}\right)}{T}\right)
$$

where $\kappa_{m}=C_{1} P_{m, 1}\left(1-P_{d, m}\right)+C_{0} P_{m, 0}\left(1-P_{f a, m}\right)$ and $q_{0} \triangleq 1$.

Proof: Before proofing Lemma 1, we note that if the SU transmits on the $m$-th channel (i.e., after $(m-1)$ times handover), the average achievable throughput is calculated as,

$$
r^{(m)}=\kappa_{m}\left(\frac{R T_{m}}{T}\right)
$$

where $R T_{m}$, the time remained for the transmission, is defined in (1).

Now, we prove the lemma using the mathematical induction. Let $\mathcal{R}_{(i)}$ denote the average achievable throughput when the maximum number of sensed channel is $i$. We intend to show that $\mathcal{R}_{(i)}$ can be computed as:

$$
\mathcal{R}_{(i)}=\sum_{m=1}^{i} q_{0} q_{1} \cdots q_{m-1} \kappa_{m}\left(\frac{R T_{m}}{T}\right)
$$

For $i=1$, the maximum achievable throughput can be calculated as [26],

$$
\mathcal{R}_{(1)}=\left(C_{1} P_{1,1}\left(1-P_{d, 1}\right)+C_{0} P_{1,0}\left(1-P_{f a, 1}\right)\right)\left(\frac{R T_{1}}{T}\right)
$$

Suppose $\mathcal{R}_{(i)}$ is true, we investigate the validity of $\mathcal{R}_{(i+1)}$. We know,

$\mathcal{R}_{(i+1)}=\mathcal{R}_{(i)}+r^{(i+1)} \times \operatorname{Pr}\{$ minimum number of HOs $=i\}$

where $r^{(i+1)}$ is defined in (47). $\operatorname{Pr}\{$ minimum number of $\mathrm{HOs}=i\}$ is equal to $q_{1} q_{2} \cdots q_{i}$, based on independency of different channels, where $q_{i}$ is defined in (2). Therefore,

$$
\mathcal{R}_{(i+1)}=\mathcal{R}_{(i)}+q_{1} q_{2} \cdots q_{i} \kappa_{i+1}\left(\frac{R T_{i+1}}{T}\right)
$$

which leads to (46) for $i=\delta$, i.e., $\mathcal{R}_{(i)}=\mathcal{R}_{(\delta)}=R$.

\section{APPENDIX B}

Suppose $P_{i, 1}^{(m)}$ denote the occupation probability of the $i$ th channel at the beginning of the $m$-th stage. For the first stage, the SUs have not sensed any channels yet, and therefore the occupation probability of each channel is equal to the corresponding PU's presence probability. Thus, we have:

$$
P_{i, 1}^{(1)}=P_{i, 1}, \quad 1 \leq i \leq N_{p}
$$

Let $\mathcal{N}_{x}$ be the number of the SUs that have requests at the node $x$. So, from Fig. 3, we have, $\mathcal{N}_{H O_{1}}=N_{s}$. The average number of the SUs that sense the $i$-th channel at the first stage, represented by $L^{(1)}$, can be computed as:

$$
L^{(1)}=\frac{p}{N_{p}} \mathcal{N}_{H O_{1}}=p \frac{N_{s}}{N_{p}}
$$

Each channel, e.g. the $i$-th channel, is sensed by $L^{(1)}$ SUs at the first stage. Each of these SUs might sense the corresponding channel free. In this case, the user starts its transmission on the channel, and therefore contributes to this channel's occupation probability at the beginning of the next sensing stage. The probability of transmission on the $i$-th channel by at least one SU conditioned on the absence of the PU can be computed as,

$$
\mathbb{U}_{i}^{(1)}=1-P_{f a, i}^{L^{(1)}}
$$

$\mathbb{U}_{i}^{(m)}$ is the probability that at least one SU transmits on the $i$-th channel (or equivalently one SU senses the channel free) at the end of the $m$-th stage conditioned on the absence of the PU. Considering (52), (53), and (54), we have:

$$
\begin{aligned}
P_{i, 1}^{(2)} & =P_{i, 1}+P_{i, 0} \mathbb{U}_{i}^{(1)} \\
& =P_{i, 1}+P_{i, 0}\left(1-P_{f a, i}^{p \frac{N_{s}}{N_{p}}}\right)
\end{aligned}
$$

At the second stage, the number of SUs whose requests enter the node $\mathrm{HO}_{2}$ is calculated in (56), where $q_{i}^{(m)}$ is defined in (31). We have,

$$
\mathbb{U}_{i}^{(2)}=1-P_{f a, i}^{L^{(2)}}
$$

where $L^{(2)}=\frac{p}{N_{p}} \mathcal{N}_{\mathrm{HO}_{2}}$. Therefore the $i$-th channel occupation probability at the beginning of the third stage can be computed as:

$$
\begin{aligned}
P_{i, 1}^{(3)} & =P_{i, 1}+P_{i, 0} \mathbb{U}_{i}^{(1)}+P_{i, 0} P_{f a, i}^{L^{(1)}} \mathbb{U}_{i}^{(2)} \\
& =P_{i, 1}^{(2)}+P_{i, 0} P_{f a, i}^{L^{(1)}} \mathbb{U}_{i}^{(2)}
\end{aligned}
$$

Following the same steps, at the $m$-th stage we have,

$$
P_{i, 1}^{(m)}=P_{i, 1}^{(m-1)}+P_{i, 0} P_{f a, i}^{L^{(1)}+L^{(2)}+\cdots+L^{(m-2)}} \mathbb{U}_{i}^{(m-1)}
$$

where

$$
\begin{aligned}
\mathbb{U}_{i}^{(m)} & =1-P_{f a, i}^{L^{(m)}} \\
L^{(m)} & =\frac{p}{N_{p}} \mathcal{N}_{H O_{m}}
\end{aligned}
$$

and

$$
\mathcal{N}_{H O_{m}}=\left((1-p)+\frac{p}{N_{p}} \sum_{i=1}^{N_{p}} q_{i}^{(m-1)}\right) \mathcal{N}_{H O_{m-1}}
$$

It is worth mentioning that for the PPRA model, a channel can be free, occupied by the PU, or occupied by some $\mathrm{SUs}^{3}$. Therefore, the SUs do not face the same received signal levels when sensing a channel at different stages. As the statistics of the signal transmitted on the channel varies at each stage, the

\footnotetext{
${ }^{3}$ The channel may have been detected as a transmission opportunity by the SUs at the previous stages
} 
$\mathcal{N}_{H O_{2}}=(1-p) \mathcal{N}_{H O_{1}}+q_{1}^{(1)} \mathcal{N}_{S_{1,1}}+q_{2}^{(1)} \mathcal{N}_{S_{1,2}}+\cdots+q_{N_{p}}^{(1)} \mathcal{N}_{S_{1, N_{p}}}=\left((1-p)+\frac{p}{N_{p}}\left(q_{1}^{(1)}+q_{2}^{(1)}+\cdots+q_{N_{p}}^{(1)}\right)\right) \mathcal{N}_{H O_{1}}$

channel sensing qualities of the SUs will change as well ${ }^{4}$. Hence, for the model to be more accurate, different miss detection probabilities in various stages for the PPRA model must be considered. On the other hand, studying this modification on the network throughput complicates the problem. In this paper, we attempt to introduce an initial version of the model, in which the applied false alarm and missed detection probabilities are related to the worst case, i.e., $P_{f a}^{\max }$ and $P_{m d}^{\max }=1-P_{d}^{\min }$. Please note that those probabilities must be considered lower than the values imposed by IEEE 802.22 standards [28]. Modifying the model for a more practical situation is left for future studies.

\section{REFERENCES}

[1] I. F. Akyildiz, W. Y. Lee, M. C. Vuran, and S. Mohanty, "NeXt generation / dynamic spectrum access / cognitive radio wireless networks: a survey," Comput. Netw. J., vol. 50, pp. 2127-2159, Sept. 2006.

[2] A. He, K. K. Bae, T. R. Newman, J. Gaeddert, K. Kim, R. Menon, L. Morales, J. Neel, Y. Zhao, J. H. Reed, and W. H. Tranter, "A survey of artificial intelligence for cognitive radios," IEEE Trans. Veh. Technol., vol. 59, no. 4, pp. 1578-1592, May 2010.

[3] L. C. Wang, C. W. Wang, and C. J. Chang, "Modeling and analysis for spectrum handoffs in cognitive radio networks," IEEE Trans. Mobile Comput., vol. 11, no. 9, pp. 1499-1513, Sept. 2012.

[4] S. Srinivasa and S. A. Jafar, "The throughput potential of cognitive radio: a theoretical perspective," IEEE Commun. Mag., pp. 73-79, May 2007.

[5] C.-W. Wang, L.-C. Wang, and F. Adachi, "Modeling and analysis for reactive-decision spectrum handoff in cognitive radio networks," 2010 IEEE Global Commun. Conf.

[6] L.-C. Wang, C.-W. Wang, and K.-T. Feng, "A queueing-theoretical framework for QoS-enhanced spectrum management in cognitive radio networks," IEEE Wireless Commun., vol. 18, no. 6, pp. 18-26, Dec. 2011.

[7] B. Kahraman and F. Buzluca, "A novel channel handover strategy to improve the throughput in cognitive radio networks," in Proc. 2011 International Wireless Commun. Mobile Comput. Conf., pp. 107-112.

[8] D. Lu, X. Huang, C. Liu, and J. Fan, "Adaptive power control based spectrum handover for cognitive radio networks," in Proc. 2010 IEEE Wireless Commun. Netw. Conf., pp. 1618-1622.

[9] S. Kim and G. B. Giannakis, "Sequential and cooperative sensing for multi-channel cognitive radios," IEEE Trans. Signal Process., vol. 58, no. 8, pp. 4239-4253, Aug. 2010.

[10] T. Zhang, K. L. Y. Wu, and D. H. K. Tsang, "Optimal scheduling of cooperative spectrum sensing in cognitive radio networks," IEEE Syst. J., vol. 4, no. 4, pp. 535-549, Dec. 2010.

[11] X. Li, Q. Zhao, X. Guan, and L. Tong, "Optimal cognitive access of Markovian channels under tight collision constraints," IEEE J. Sel. Areas Commun., vol. 29, no. 4, pp. 476-759, Apr. 2011.

[12] A. Sabharwal, A. Khoshnevis, and E. Knightly, "Opportunistic spectral usage: bounds and a multi-band CSMA/CA protocol," IEEE/ACM Trans. Netw., vol. 15, no. 3, pp. 533-545, June 2007.

[13] J. Jia, Q. Zhang, and X. S. Shen, "HC-MAC: a hardware-constrained cognitive MAC for efficient spectrum management," IEEE J. Sel. Areas Commun., vol. 26, no. 1, pp. 106-117, June 2008.

[14] D. J. Lee and M. S. Jang, "Optimal spectrum sensing time considering spectrum handoff due to false alarm in cognitive radio networks," IEEE Commun. Lett., vol. 13, no. 12, pp. 899-901, Dec. 2009.

${ }^{4}$ Please note that a false alarm occurs when a free channel is mistakenly sensed busy. Consequently, there are no PU or SUs signals on the channel when a false alarm happens. Therefore, the possible changes in the level of the signals in various stages do not affect the false alarm probability. Instead, the miss detection probability is directly related to the received SNR and may change in different stages, depending on the number of SUs transmit on the corresponding channel.
[15] H. Jiang, R. F. L. Lai, and H. V. Poor, "Optimal selection of channel sensing order in cognitive radio," IEEE Trans. Wireless Commun., vol. 8, no. 1, pp. 297-307, Jan. 2009.

[16] R. Fan and H. Jiang, "Channel sensing-order setting in cognitive radio networks: a two-user case," IEEE Trans. Veh. Technol., vol. 58, no. 9, pp. 4997-5008, Nov. 2009.

[17] H. Shokri-Ghadikolaei and M. Nasiri-Kenari, "Sensing matrix setting schemes for cognitive networks and their performance analysis," IET Commun., vol. 6, no. 17, pp. 3026-3035, Nov. 2012.

[18] H. Shokri-Ghadikolaei and R. Fallahi, "Intelligent sensing matrix setting scheme in cognitive networks," IEEE Commun. Lett., vol. 16, no. 11, pp. 1824-1827, Nov. 2012.

[19] W. Gabran, P. Pawelczak, and D. Cabric, "Throughput and collision analysis of multichannel multistage spectrum sensing algorithms," IEEE Trans. Veh. Technol., vol. 60, no. 7, pp. 3309-3329, Sept. 2011.

[20] J. Park, P. Pawelczak, and D. Cabric, "Performance of joint spectrum sensing and MAC algorithms for a multichannel opportunistic spectrum access ad hoc networks," IEEE Trans. Mobile Comput., vol. 10, no. 7, pp. 1011-1027, July 2011.

[21] L. Lai, H. E. Gamal, H. Jiang, and H. V. Poor, "Optimal medium access control in cognitive radios: a sequential design approach," in Proc. 2008 IEEE ICASSP, pp. 2073-2076.

[22] — , "Cognitive medium access: exploration, exploitation, and competition," IEEE Trans. Mobile Comput., vol. 10, no. 2, pp. 239-253, Feb. 2011.

[23] Y.-C. Liang, K.-C. Chen, G. Y. Li, and P. Mahonen, "Cognitive radio networking and communications: an overview," IEEE Trans. Veh. Technol., vol. 60, no. 7, pp. 3386-3407, Sept. 2011.

[24] B. F. Lo, "A survey of common control channel design in cognitive radio networks," Physical Commun., vol. 4, no. 1, pp. 26-39, Mar. 2011.

[25] X. Chio, M. Miyazawa, and M. Pinedo, Queueing Networks. John Wiley \& Sons, 1999.

[26] Y. C. Liang, Y. Zeng, E. C. Y. Peh, and A. T. Hoang, "Sensingthroughput tradeoff for cognitive radio networks," IEEE Trans. Wireless Commun., vol. 7, no. 4, pp. 1326-1337, Apr. 2008.

[27] R. Fan and H. Jiang, "Optimal multi-channel cooperative sensing in cognitive radio networks," IEEE Trans. Wireless Commun., vol. 9, no. 3, pp. 1128-1138, Mar. 2010.

[28] C. R. Stevenson, G. Chouinard, W. H. Z. Lei, and S. J. Shellhammer, "IEEE 802.22: the first cognitive radio wireless regional area network standard," IEEE Commun. Mag., vol. 47, no. 1, pp. 130-138, Jan. 2009.

[29] Y. C. Tay, K. Jamieson, and H. Balakrishnan, "Collision-minimizing CSMA and its applications to wireless sensor networks," IEEE Trans. Consum. Electron., vol. 22, no. 6, pp. 1048-1057, Aug. 2004.

[30] R. Bruno, M. Conti, and E. Gregori, "Optimization of efficiency and energy consumption in p-persistent CSMA-based wireless LAN," IEEE Trans. Mobile Comput., vol. 1, no. 1, pp. 10-31, Jan. 2002.

[31] S. Zheng, Y.-C. Liang, P. Y. Kam, and A. T. Hoang, "Cross-layered design of spectrum sensing and MAC for opportunistic spectrum access," in Proc. 2009 IEEE WCNC, pp. 1-6.

[32] S. H. Park, H. Yoon, and J. W. Kim, "A cross-layered network adaptive $\mathrm{HD}$ video steaming in digital A/V home network: channel monitoring and video rate adaptation," IEEE Trans. Consum. Electron., vol. 52, no. 4, pp. 1245-1252, Nov. 2006.

[33] P. Pawelczak, K. Nolan, L. Doyle, S. W. Oh, and D. Cabric, "Cognitive radio: ten years of experimentation and development," IEEE Commun. Mag., pp. 90-100, Mar. 2011.

[34] T. Ycek and H. Arslan, "A survey of spectrum sensing algorithms for cognitive radio applications," IEEE Commun. Surveys \& Tutorials, vol. 11, no. 1, pp. 116-160, May 2009.

[35] "Draft standard for wireless regional area networks part 22: cognitive wireless RAN medium access control (MAC) and physical layer (PHY) specifications," IEEE 802.22 Working Group. 


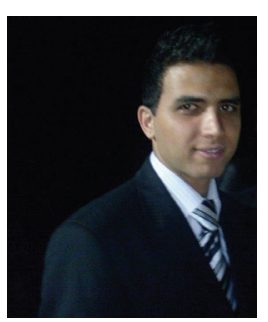

Hossein Shokri-Ghadikolaei received his B.S. and M.S. degrees in communication systems from Iran University of Science and Technology and Sharif University of Technology, Tehran, Iran, in 2009 and 2011, respectively. He is currently with the Communication Technology Institute of Iran Telecommunication Research Center (ITRC), Tehran, Iran. His research interests include cognitive radio networks, heterogenous wireless networks, and LTE-Advanced systems.

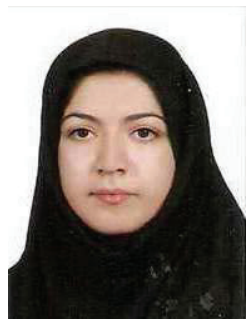

Fatemeh Sheikholeslami received her B.S. and M.S. degrees in communication systems from University of Tehran and Sharif University of Technology, Tehran, Iran, in 2010 and 2012, respectively. She is currently with the Communication Technology Institute of Iran, Telecommunication Research Center (ITRC), Tehran, Iran. Her research interests include cognitive radio networks and queuing systems.
Masoumeh Nasiri-Kenari (S90-M1994) received the B.S and M.S. degrees in electrical engineering from Isfahan University of Technology, Isfahan, Iran, in 1986 and 1987, respectively, and the Ph.D. degree in electrical engineering from the University of Utah, Salt Lake City, in 1993. From 1987 to 1988, she was a Technical Instructor and Research Assistant at Isfahan University of Technology. Since 1994, she has been with the Department of Electrical Engineering, Sharif University of Technology, Tehran, Iran, where she is now a Professor. Dr. Nasiri-Kenari also serves as the head of Wireless Lab. of Advanced Communications Research Institute, Sharif University of Technology. From 1999-2001, She was a Co-Director of the Advanced Communication Science Research Laboratory, Iran Telecommunication Research Center, Tehran, Iran. Her current research interests are in wireless communication systems, error correcting codes, and optical communication systems. 